\title{
FINITE SPEED OF PROPAGATION AND WAITING TIME PHENOMENA FOR DEGENERATE PARABOLIC EQUATIONS WITH LINEAR GROWTH LAGRANGIAN*
}

\author{
LORENZO GIACOMELLI ${ }^{\dagger}$
}

\begin{abstract}
We consider a class of degenerate parabolic equations with linear growth Lagrangian. Two prototypes within this class, sharing common features with nonlinear transport equations, are the relativistic porous medium equation and the speed-limited (or flux-limited) porous medium equation. In arbitrary space dimension, we prove that entropy solutions to the Cauchy problem satisfy the finite speed of propagation property. For the two aforementioned prototypes, we provide a condition on the growth of the initial datum which guarantees the occurrence of a waiting time phenomenon; we also present a heuristic argument in favor of the optimality of such condition.
\end{abstract}

Key words. degenerate parabolic equations, singular parabolic equations, nonlinear transport equations, entropy solutions, finite speed of propagation, waiting time phenomena

AMS subject classifications. 35K65, 35K67, 35L03, 35D99, 35B99

DOI. $10.1137 / 130945077$

1. Introduction. Generally speaking, degenerate parabolic equations with linear growth Lagrangian are a class of second-order diffusion equations of the form

$$
u_{t}=\operatorname{div} \mathbf{a}(u, \nabla u)
$$

(plus lower-order terms) which display both a degeneracy with respect to $u$, in the sense that

$$
\lim _{z \rightarrow 0^{+}} \mathbf{a}(z, \mathbf{v})=\mathbf{0} \text { for all } \mathbf{v} \in \mathbb{R}^{N},
$$

and a linear growth of the associated Lagrangian, in the sense that

$$
\frac{1}{|\mathbf{v}|} \lim _{t \rightarrow+\infty} \mathbf{a}(z, t \mathbf{v}) \cdot \mathbf{v}=: \varphi(z) \quad \text { for all } z \geq 0 .
$$

While referring to $[4,9,11,12]$ for thorough referenced discussions on the models and the theory for this class, here we just point out two prototypes: the relativistic porous medium equation,

$$
u_{t}=\nu \operatorname{div}\left(\frac{u^{m} \nabla u}{\sqrt{u^{2}+\nu^{2} c^{-2}|\nabla u|^{2}}}\right), \quad m \in(1,+\infty),
$$

which generalizes the so-called relativistic heat equation $(m=1)$, and the speedlimited porous medium equation,

$$
u_{t}=\nu \operatorname{div}\left(\frac{u \nabla u^{M-1}}{\sqrt{1+\nu^{2} c^{-2}\left|\nabla u^{M-1}\right|^{2}}}\right), \quad M \in(1,+\infty),
$$

\footnotetext{
${ }^{*}$ Received by the editors November 12, 2013; accepted for publication (in revised form) April 14, 2015; published electronically June 18, 2015.

http://www.siam.org/journals/sima/47-3/94507.html

†SBAI Department, Sapienza University of Rome, 16, 00161 Rome, Italy (lorenzo.giacomelli@ sbai.uniroma1.it).
} 
which has been recently proposed in [12] (under the name of "flux-limited porous medium equation"). As $c \rightarrow+\infty$, both (1.3) and (1.4) (with $m=M$ and up to a factor $M-1$ ) converge to the porous medium equation,

$$
u_{t}=\nu \operatorname{div}\left(u^{m-1} \nabla u\right)
$$

(see [13] for rigorous convergence results). Up to the scaling $\hat{t}=\frac{c^{2}}{\nu} t, \hat{x}=\frac{c}{\nu} x$, we assume without losing generality that

$$
\nu=c=1 .
$$

A mathematical theory for suitable classes of equations of the form (1.1)-(1.2) has been recently developed, based on the concept of entropy solutions (we refer to section 2 for a brief introduction to this concept and its precise definition). This note is concerned with two qualitative properties of entropy solutions to the Cauchy problem for (1.1) and (1.3)-(1.4), respectively: the finite speed of propagation property, which means that the solutions' support remains bounded for positive times provided it is initially so, and the waiting time phenomenon, which means that, under suitable growth conditions on the initial data, a positive time exists during which the solutions' support locally does not expand.

The finite speed of propagation property was proved in [5] for entropy solutions to (1.3) with $m=1$, the speed being bounded above by 1 (recall (1.5)). For (1.3) and (1.4), conjectures have been recently formulated in [8]. Here, by a generalization of the arguments in [5], we confirm these conjectures: If $u_{0} \in L^{\infty}\left(\mathbb{R}^{N}\right)$ is nonnegative with compact support, and $u$ is the entropy solution to the Cauchy problem for (1.3) or (1.4) with initial datum $u_{0}$ (see Remark 2.3), then

$$
\begin{aligned}
& \operatorname{supp}(u(t)) \subseteq \operatorname{supp}\left(u_{0}\right)+\overline{B\left(0, m\left\|u_{0}\right\|_{\infty}^{m-1} t\right)} \text { if } u \text { solves (1.3) or } \\
& \operatorname{supp}(u(t)) \subseteq \operatorname{supp}\left(u_{0}\right)+\overline{B(0, t)} \text { if } u \text { solves }(1.4)
\end{aligned}
$$

for all $t>0$, where + denotes the Minkowski addition of two sets. Formally, writing (1.3) and (1.4) in the form $u_{t}=\operatorname{div}(u \mathbf{v})$ and bounding $|\mathbf{v}|$ from above, one finds $|\mathbf{v}| \leq u^{m-1}$ for (1.3) and $|\mathbf{v}| \leq 1$ for (1.4). Hence we expect that (1.7) is optimal, whereas (1.6) may overestimate the speed by a factor $m$.

As discussed in Remark 1.3, both (1.6) and (1.7) are immediate consequences of a more general result, which extends [5, Theorem 4] and which we now introduce (a related extension has been independently obtained in [7]). We assume the following.

Assumption 1.1. Let $Q=(0, \infty) \times \mathbb{R}^{N}$. The function a $: \bar{Q} \rightarrow \mathbb{R}^{N}$ is such that

(i) (Lagrangian) there exists $f \in C(\bar{Q})$ such that $\nabla_{\mathbf{v}} f=\mathbf{a} \in C(\bar{Q}), f(z, \cdot)$ is convex, $f(z, 0)=0$ for all $z \in[0, \infty)$, and

$$
C_{0}(z)|\mathbf{v}|-D_{0}(z) \leq f(z, \mathbf{v}) \leq M_{0}(z)(1+|\mathbf{v}|) \text { for all }(z, \mathbf{v}) \in Q
$$

for nonnegative continuous functions $M_{0}, C_{0} \in C([0, \infty))$ and $D_{0} \in C((0, \infty))$, with $C_{0}(z)>0$ for $z>0$

(ii) $($ flux $) D_{\mathbf{v}} \mathbf{a} \in C(\bar{Q}) ; \mathbf{a}(z, 0)=\mathbf{a}(0, \mathbf{v})=0$ and $h(z, \mathbf{v}):=\mathbf{a}(z, \mathbf{v}) \cdot \mathbf{v}=h(z,-\mathbf{v})$ for all $(z, \mathbf{v}) \in \bar{Q}$; for any $R>0$ there exists $M_{R}>0$ such that

$$
|\mathbf{a}(z, \mathbf{v})-\mathbf{a}(\hat{z}, \mathbf{v})| \leq M_{R}|z-\hat{z}| \quad \text { for all } z, \hat{z} \in[0, R] \text { and all } \mathbf{v} \in \mathbb{R}^{N} ;
$$

(iii) (recession functions) the recession functions $f^{0}$ and $h^{0}$, defined by

$$
f^{0}(z, \mathbf{v})=\lim _{t \rightarrow+\infty} \frac{1}{t} f(z, t \mathbf{v}), \quad h^{0}(z, \mathbf{v})=\lim _{t \rightarrow+\infty} \frac{1}{t} h(z, t \mathbf{v}),
$$


exist in $\bar{Q}$ and $f^{0} \equiv h^{0}$; furthermore, a function $\varphi \in \operatorname{Lip}_{l o c}([0, \infty))$, with $\varphi(0)=0$ and $\varphi>0$ in $(0, \infty)$, exists such that

$$
f^{0}(z, \mathbf{v})=h^{0}(z, \mathbf{v})=\varphi(z)|\mathbf{v}| \quad \text { for all }(z, \mathbf{v}) \in \bar{Q}
$$

(cf. (1.2)), and $\mathbf{a}(z, \mathbf{w}) \cdot \mathbf{v} \leq \varphi(z)|\mathbf{v}|$ for all $(z, \mathbf{v}) \in Q, \mathbf{w} \in \mathbb{R}^{N}$.

We prove the following.

THEOREM 1.2. Let a be such that Assumption 1.1 holds. Let $u_{0} \in L^{\infty}\left(\mathbb{R}^{N}\right)$ be nonnegative with compact support and let $u$ be an entropy solution to the Cauchy problem for (1.1) with initial datum $u_{0}$ in the sense of Definition 2.1. Then

$$
\operatorname{supp}(u(t)) \subseteq \operatorname{supp}\left(u_{0}\right)+\overline{B(0, V t)}, \quad V:=\operatorname{ess~sup}_{z \in\left(0,\left\|u_{0}\right\|_{\infty}\right)} \varphi^{\prime}(z)
$$

for all $t>0$, where $\varphi$ is defined in Assumption 1.1(iii).

Remark 1.3. It is not difficult to check that, in the particular cases of (1.3) and (1.4), the corresponding fluxes,

$$
\mathbf{a}_{1}(z, \mathbf{v})= \begin{cases}\frac{z^{m} \mathbf{v}}{\sqrt{z^{2}+|\mathbf{v}|^{2}}} & \text { if }(z, \mathbf{v}) \neq(0,0) \\ 0 & \text { if }(z, \mathbf{v})=(0,0)\end{cases}
$$

and

$$
\mathbf{a}_{2}(z, \mathbf{v})= \begin{cases}\frac{(M-1) z^{M-1} \mathbf{v}}{\sqrt{1+(M-1)^{2} z^{2 M-4}|\mathbf{v}|^{2}}} & \text { if } z>0 \\ 0 & \text { if } z=0\end{cases}
$$

satisfy Assumption 1.1 with

$$
f_{1}(z, \mathbf{v})=z^{m}\left(\sqrt{z^{2}+|\mathbf{v}|^{2}}-z\right)
$$

respectively,

$$
f_{2}(z, \mathbf{v})= \begin{cases}\frac{z^{3-M}}{M-1}\left(\sqrt{1+(M-1)^{2} z^{2 M-4}|\mathbf{v}|^{2}}-1\right) & \text { if } z>0, \\ 0 & \text { if } z=0 .\end{cases}
$$

Therefore, (1.6) and (1.7) follow immediately from

$$
\varphi(z)=\frac{1}{|\mathbf{v}|} \lim _{t \rightarrow+\infty} \mathbf{a}(z, t \mathbf{v}) \cdot \mathbf{v}= \begin{cases}z^{m} & \text { for }(1.3), \\ z & \text { for (1.4). }\end{cases}
$$

The waiting time phenomenon for (1.3) has been recently discussed in $[6$, section $5]$ and [9, section 4.2.1] via numerical and formal arguments. In the present note we give sufficient conditions for the occurrence of a waiting time phenomenon.

TheOREM 1.4. Let $u_{0} \in L^{\infty}\left(\mathbb{R}^{N}\right)$ be nonnegative with compact support, $x_{0} \in \mathbb{R}^{N}$, and let $u$ be the entropy solution to the Cauchy problem for (1.3) or (1.4) with initial datum $u_{0}$ in the sense of Definition 2.1. Positive constants $A_{m}$ (resp., $A_{M}$ ), depending only on $N$ and $m$ (resp., $M$ ), exists such that if

$$
\begin{aligned}
& \operatorname{ess\operatorname {sup}}\left|x-x_{0}\right|^{-\frac{1}{m-1}} u_{0}(x)=C<+\infty \quad \text { if } u \text { solves (1.3) or } \\
& \text { ess sup }\left|x-x_{0}\right|^{-\frac{2}{M-1}} u_{0}(x)=C<+\infty \quad \text { if } u \text { solves }(1.4),
\end{aligned}
$$


then

$$
\operatorname{essips}_{x \in \mathbb{R}^{N}}\left|x-x_{0}\right|^{-\frac{1}{m-1}} u(t, x) \leq C\left(\frac{\tau_{*}-t}{\tau_{*}}\right)^{-\frac{1}{m-1}} \quad \text { for all } t<\tau_{*}:=\left(C / A_{m}\right)^{1-m}
$$

if $u$ solves (1.3) or

$$
\underset{x \in \mathbb{R}^{N}}{\operatorname{ess} \sup _{0}}\left|x-x_{0}\right|^{-\frac{2}{M-1}} u(t, x) \leq C\left(\frac{\tau_{*}-t}{\tau_{*}}\right)^{-\frac{1}{M-1}} \quad \text { for all } t<\tau_{*}:=\left(C / A_{M}\right)^{1-M}
$$

if $u$ solves (1.4).

Generally speaking, Theorem 1.4 says that if $u_{0}$ decays sufficiently fast to zero at $x_{0}$, then $u\left(t, x_{0}\right)=0$ for all $t<\tau_{*}$. A slightly different formulation of the waiting time phenomenon, involving an exterior cone property of $\operatorname{supp}\left(u_{0}\right)$ at $x_{0}$, has been adopted in $[15,20,21]$ for a rather general class of degenerate parabolic equations (see [19] for a recent referenced discussion on this subject). The next result shows that such formulation is implied by Theorem 1.4 .

COROLLARY 1.5. Under the assumptions of Theorem 1.4, assume in addition that $\operatorname{supp}\left(u_{0}\right)$ has the exterior cone property at $x_{0} \in \partial \operatorname{supp}\left(u_{0}\right)$, i.e., $B_{0} \geq 0$ and $w \in \mathbb{R}^{N},|w|=1$, exist such that $\operatorname{supp}\left(u_{0}\right) \cap \mathcal{C}_{B_{0}}=\emptyset$, where

$$
\mathcal{C}_{B_{0}}:=\left\{x \in \mathbb{R}^{N}:\left(x_{0}-x\right) \cdot w>B_{0}\left|\left(x_{0}-x\right)-\left(\left(x_{0}-x\right) \cdot w\right) w\right|\right\} .
$$

Then positive constants $A_{m, 1}$ (resp., $A_{M, 1}$ ), depending on $N, B_{0}$, and $m$ (resp., $M)$, exist such that $x_{0} \in \overline{\mathbb{R}^{N} \backslash \operatorname{supp}(u(t, \cdot))}$ for all $t<\left(C / A_{m, 1}\right)^{1-m}$ (resp., $t<$ $\left.\left(C / A_{M, 1}\right)^{1-M}\right)$.

The exponents $1 /(m-1)$ and $2 /(M-1)$ in (1.15)-(1.16) are critical in our argument. A natural question is whether this is a technical or a substantial constraint. In one space dimension we present a heuristic argument, based on the hyperbolic character of the equation near $x_{0}$, which in fact suggests that the critical exponents are optimal, in the sense that no waiting time phenomenon occurs if $\operatorname{supp}\left(u_{0}\right) \subset\left[x_{0}, \infty\right)$ and

$$
\begin{aligned}
& \lim _{x \rightarrow x_{0}^{+}}\left(x-x_{0}\right)^{-\frac{1}{m-1}} u_{0}(x)=+\infty \quad \text { if } u \text { solves }(1.3) \text { or } \\
& \lim _{x \rightarrow x_{0}^{+}}\left(x-x_{0}\right)^{-\frac{2}{M-1}} u_{0}(x)=+\infty \quad \text { if } u \text { solves }(1.4) .
\end{aligned}
$$

For (1.3), our heuristic argument also suggests that, when (1.19) is violated, a jump instantaneously starts developing at the left boundary of the support, $s_{-}(t)\left(s_{-}(0)=\right.$ $\left.x_{0}\right)$, causing its expansion. Scaling laws for both $s_{-}(t)$ and the height of the jump at that point may then be obtained using the method of characteristics and the RankineHugoniot condition: if $u_{0}(x) \sim\left(x-x_{0}\right)_{+}^{\alpha}$ as $x \rightarrow x_{0}$, then (see (5.9) and (5.10) below)

$$
s_{-}(t)-x_{0} \sim-t^{\frac{1}{1-\alpha(m-1)}} \quad \text { and } \quad u_{+}\left(t, s_{-}(t)\right) \sim t^{\frac{\alpha}{1-\alpha(m-1)}} \quad \text { for } t \ll 1 \text {. }
$$

Our heuristic seems to lead to different conclusions with respect to the numerical and formal arguments in [9, section 4.2.1], where another critical exponent, $1 / m$, is predicted for (1.3) (see Remark 5.1). Such discrepancy points toward the necessity of sharp results on the nonoccurrence of a waiting time phenomenon, possibly through the construction of suitable classes of subsolutions whose support instantaneously expands. 
The argument for Theorem 1.4 is based on comparison. Therefore, another natural question is whether known energy methods, developed in recent years to study the waiting time phenomenon for degenerate parabolic equations and systems (also of higher order; see $[15,19,20,21])$, may be adapted to cover the present class. This would lead to general results for (1.1) rather than for specific models such as (1.3) and (1.4), as well as to weaker, integral-type conditions on the initial datum, in the spirit of those obtained in [1] for the porous medium equation: e.g., we expect that (1.15) may be replaced by $\sup _{r>0} r^{-\frac{1}{m-1}-N} \int_{B_{r}\left(x_{0}\right)} u_{0}(x) \mathrm{d} x<+\infty$.

The note is organized as follows. In section 2 we review the concept of entropy (super)solution and (slight generalizations of) basic known results; in section 3 we prove Theorem 1.2; in section 4 we prove Theorem 1.4 and Corollary 1.5; finally, in section 5 we present the heuristic argument in favor of the optimality of the exponents in (1.15) and (1.16).

2. Entropy (super)solutions. The concept of entropy solutions to the Cauchy problem for (1.1) was introduced in [3] and later extended in [10,12]. At the core of this concept is an entropy inequality (cf. (2.8) below) that we now introduce.

For $a, b, \ell \in \mathbb{R}$ we let

$$
T_{a, b}^{\ell}(r)=\max \{\min \{b, r\}, a\}-\ell
$$

We define the sets

$$
\mathcal{T}^{+}=\left\{T_{a, b}^{\ell}: 0<a<b, \ell \leq a\right\}, \quad \mathcal{T}^{-}=\left\{T_{a, b}^{\ell}: 0<a<b, \ell \geq b\right\} .
$$

We also use the following notation:

$$
\text { given } T=T_{a, b}^{\ell} \in \mathcal{T}^{+} \cup \mathcal{T}^{-} \text {, we let } T^{0}:=T+\ell=T_{a, b}^{0} \text {. }
$$

For $f \in L_{l o c}^{1}(\mathbb{R})$ we let

$$
J_{f}(r):=\int_{0}^{r} f(s) \mathrm{d} s .
$$

Let $S, T \in \mathcal{T}^{+} \cup \mathcal{T}^{-}$. Formally testing (1.1) by $T(u) S(u) \phi$, with $\phi \in C_{c}^{\infty}\left((0, \infty) \times \mathbb{R}^{N}\right)$, after integration by parts we see that

$$
\begin{aligned}
& \int_{0}^{+\infty} \int_{\mathbb{R}^{N}} J_{T S}(u) \phi_{t} \mathrm{~d} x \mathrm{~d} t=\int_{0}^{+\infty} \int_{\mathbb{R}^{N}} T(u) S(u) \mathbf{a}(u, \nabla u) \cdot \nabla \phi \mathrm{d} x \mathrm{~d} t \\
& \quad+\int_{0}^{+\infty} \int_{\mathbb{R}^{N}} \phi(S(u) \mathbf{a}(u, \nabla u) \cdot \nabla T(u)+T(u) \mathbf{a}(u, \nabla u) \cdot \nabla S(u)) \mathrm{d} x \mathrm{~d} t .
\end{aligned}
$$

Since $\nabla S(u)=\nabla S^{0}(u)$ and $u=S^{0}(u)$ on $\operatorname{supp}\left(\nabla S^{0}(u)\right)$, we have

$$
\begin{aligned}
T(u) \mathbf{a}(u, \nabla u) \cdot \nabla S(u) & =T(u) \mathbf{a}(u, \nabla u) \cdot \nabla S^{0}(u) \\
& =T\left(S^{0}(u)\right) \mathbf{a}\left(S^{0}(u), \nabla S^{0}(u)\right) \cdot \nabla S^{0}(u) \\
& =T\left(S^{0}(u)\right) h\left(S^{0}(u), \nabla S^{0}(u)\right),
\end{aligned}
$$

where $h$ is defined in Assumption 1.1(ii). Analogously,

$$
S(u) a(u, \nabla u) \cdot \nabla T(u)=S\left(T^{0}(u)\right) h\left(T^{0}(u), \nabla T^{0}(u)\right) .
$$


In view of (2.3)-(2.4), (2.2) may be rewritten as

$$
\begin{aligned}
\int_{0}^{+\infty} & \int_{\mathbb{R}^{N}} \phi\left(S\left(T^{0}(u)\right) h\left(T^{0}(u), \nabla T^{0}(u)\right)+T\left(S^{0}(u)\right) h\left(S^{0}(u), \nabla S^{0}(u)\right)\right) \mathrm{d} x \mathrm{~d} t \\
= & \int_{0}^{+\infty} \int_{\mathbb{R}^{N}}\left(J_{T S}(u) \phi_{t}-T(u) S(u) \mathbf{a}(u, \nabla u) \cdot \nabla \phi\right) \mathrm{d} x \mathrm{~d} t .
\end{aligned}
$$

On passing from formal to rigorous arguments, e.g., when constructing a solution to (1.1) as the limit of solutions to suitable approximating problems, one needs to argue by lower semicontinuity on the left-hand side of (2.5). It turns out ([16] ; see also the discussion in $\left[3\right.$, sections 2.2 and 3.2]) that, for any nonnegative $\phi \in C_{c}\left(\mathbb{R}^{N}\right)$, any $T \in \mathcal{T}^{+} \cup \mathcal{T}^{-}$, and any $S \in \operatorname{Lip}\left(\mathbb{R} ;[0, \infty)\right.$ ) with $S^{\prime}$ compactly supported (in particular, any $S \in \mathcal{T}^{+}$),

$$
\begin{aligned}
\mathcal{R}(\phi S h, T)(u) & :=\int_{\mathbb{R}^{N}} \phi S\left(T^{0}(u)\right) h\left(T^{0}(u), \nabla T^{0}(u)\right) \mathrm{d} x+\int_{\mathbb{R}^{N}} \phi \mathrm{d}\left|D^{s} J_{S \varphi}\left(T^{0}(u)\right)\right| \\
& \leq \liminf _{n \rightarrow \infty} \int_{\mathbb{R}^{N}} \phi S\left(T^{0}\left(u_{n}\right)\right) h\left(T^{0}\left(u_{n}\right), \nabla T^{0}\left(u_{n}\right)\right) \mathrm{d} x
\end{aligned}
$$

for any $u \in B V\left(\mathbb{R}^{N}\right)$ and any $\left\{u_{n}\right\} \subset W^{1,1}\left(\mathbb{R}^{N}\right)$ such that $u_{n} \rightarrow u$ in $L^{1}\left(\mathbb{R}^{N}\right)$, where $\varphi$ is given by (1.8). (We use standard notation and concepts for $B V$ functions [2]. In particular, for $u \in B V\left(\mathbb{R}^{N}\right), \nabla u \mathcal{L}^{N}$, resp., $D^{s} u$, denote the absolutely continuous, resp., singular, parts of $D u$ with respect to the Lebesgue measure $\mathcal{L}^{N}$.) Note that under standard continuity and coercivity assumptions, when $\Omega \subset \mathbb{R}^{N}$ is a bounded open set $\mathcal{R}\left(\chi_{\Omega} S h, T\right)(u)$ coincides with the relaxation of the right-hand side of (2.6), i.e.,

$$
\mathcal{R}\left(\chi_{\Omega} S h, T\right)(u)=\inf \left\{\liminf _{n \rightarrow \infty} \int_{\Omega} S\left(T^{0}\left(u_{n}\right)\right) h\left(T^{0}\left(u_{n}\right), \nabla T^{0}\left(u_{n}\right)\right) \mathrm{d} x\right\}
$$

for any $u \in B V(\Omega)$, where the infimum is taken among all $\left\{u_{n}\right\} \subset W^{1,1}(\Omega)$ such that $u_{n} \rightarrow u$ in $L^{1}(\Omega)$ (see, e.g., [14]).

We denote by $h_{S}(u, D T(u))$ the Radon measure defined by

$$
\left\langle h_{S}(u, D T(u)), \phi\right\rangle:=\mathcal{R}(\phi S h, T)(u) \quad \text { for all } \phi \in C_{c}\left(\mathbb{R}^{N}\right) .
$$

Combining (2.5)-(2.7) yields the following entropy inequality:

$$
\begin{aligned}
& \int_{0}^{+\infty}\left\langle h_{S}(u, D T(u))+h_{T}(u, D S(u)), \phi\right\rangle \mathrm{d} t \\
& \leq \int_{0}^{+\infty} \int_{\mathbb{R}^{N}}\left(J_{T S}(u) \phi_{t}-T(u) S(u) \mathbf{a}(u, \nabla u) \cdot \nabla \phi\right) \mathrm{d} x \mathrm{~d} t .
\end{aligned}
$$

This motivates the crucial part, (iv), of the definition of entropy solutions.

Definition 2.1. Let $u_{0} \in L^{\infty}\left(\mathbb{R}^{N}\right) \cap L^{1}\left(\mathbb{R}^{N}\right)$ nonnegative. A nonnegative function $u \in C\left([0,+\infty) ; L^{1}\left(\mathbb{R}^{N}\right)\right) \cap L^{\infty}\left((0, \infty) \times \mathbb{R}^{N}\right)$ is an entropy solution to the Cauchy problem for (1.1) with initial datum $u_{0}$ if $u(0)=u_{0}$ and

(i) $T_{a, b}^{a}(u) \in L_{l o c}^{1}\left((0,+\infty) ; B V\left(\mathbb{R}^{N}\right)\right)$ for all $0<a<b$;

(ii) $\mathbf{a}(u, \nabla u) \in L_{l o c}^{\infty}\left([0, \infty) \times \mathbb{R}^{N}\right)$;

(iii) $u_{t}=\operatorname{div}(\mathbf{a}(u, \nabla u))$ in the sense of distributions; 
(iv) inequality (2.8) holds for any $S, T \in \mathcal{T}^{+}$and any nonnegative smooth function $\phi$ with compact support in $(0,+\infty) \times \mathbb{R}^{N}$.

The following holds.

THEOREM 2.2. Let a be such that Assumption 1.1 holds. For any nonnegative $u_{0} \in L^{\infty}\left(\mathbb{R}^{N}\right) \cap L^{1}\left(\mathbb{R}^{N}\right)$ there exists at most one entropy solution to the Cauchy problem for (1.1) with initial datum $u_{0}$. In addition, if either

(H1) $D_{0} \in C([0, \infty))$ and $A, B \geq 0, \alpha, \beta \geq 1$ exist such that $D_{0}(z) \leq A z^{\alpha}+B z^{\beta}$ for all $z \geq 0$,

or

(H2) for any $R>0$ there exists $c_{R}>0$ such that $C_{0}(z) \geq c_{R} z$ and $\varphi(z) \geq c_{R} z$ for all $z \in[0, R]$,

then the entropy solution exists.

Remark 2.3. Theorem 2.2 is contained in, or follows from, earlier results in $[3,12]$ : we refer to the appendix for details. We note that the flux $\mathbf{a}_{1}$ in (1.10) satisfies (H1) with $D_{0}(z)=z^{m+1}$ (cf. (1.12)) and that the flux $\mathbf{a}_{2}$ in (1.11) satisfies (H2) with $C_{0}(z)=z($ cf. (1.13)-(1.14)). Hence Theorem 2.2 applies in particular to (1.3) and (1.4).

The formal arguments behind the concept of supersolutions [5, Definition 5] are analogous to the ones above, except that $S \in \mathcal{T}^{-}$: on defining $h_{S}(u, D T(u))=$ $-h_{(-S)}(u, D T(u))$ for $S \in \mathcal{T}^{-}$and $T \in \mathcal{T}^{+} \cup \mathcal{T}^{-}$, they lead to the same inequality, (2.8). In fact, in dealing with the waiting time phenomenon we need a slight extension of $\left[5\right.$, Definition 5], which does not require uniform boundedness in $\mathbb{R}^{N}$.

Definition 2.4. Let $\tau>0$. A nonnegative function $u \in C\left([0, \tau) ; L_{\text {loc }}^{1}\left(\mathbb{R}^{N}\right)\right) \cap$ $L_{\text {loc }}^{\infty}\left([0, \tau] \times \mathbb{R}^{N}\right)$ is an entropy supersolution to $(1.1)$ in $(0, \tau) \times \mathbb{R}^{N}$ if

(i) $T_{a, b}^{a}(u) \in L_{l o c}^{1}\left((0, \tau) ; B V_{l o c}\left(\mathbb{R}^{N}\right)\right)$ for all $0<a<b$;

(ii) $\mathbf{a}(u(t), \nabla u(t)) \in L_{\text {loc }}^{\infty}\left(\mathbb{R}^{N}\right)$ for a.e. $t \in(0, \tau)$;

(iii) (2.8) holds for any $S \in \mathcal{T}^{-}, T \in \mathcal{T}^{+}$, and any nonnegative smooth function $\phi$ with compact support in $(0, \tau) \times \mathbb{R}^{N}$.

The following result generalizes [5, Theorem 2].

THEOREM 2.5. Let a be such that Assumption 1.1 holds. Let $u$ be an entropy solution to the Cauchy problem for (1.1) with initial datum $u_{0} \in L^{\infty}\left(\mathbb{R}^{N}\right) \cap L^{1}\left(\mathbb{R}^{N}\right)$ and $\bar{u}$ be an entropy supersolution to $(1.1)$ in $(0, \tau)$ with $\bar{u}(0) \in L_{\text {loc }}^{\infty}\left(\mathbb{R}^{N}\right)$. If $\bar{u}(0) \geq u_{0}$ and either

(a) $\bar{u}(t) \in B V\left(\mathbb{R}^{N}\right)$ for a.e. $t \in(0, \tau), \bar{u} \in C\left([0, \tau) ; L^{1}\left(\mathbb{R}^{N}\right)\right), T_{a, b}^{a}(\bar{u}) \in L^{1}((0, \tau)$; $\left.B V\left(\mathbb{R}^{N}\right)\right)$ for all $0<a<b$, and $\mathbf{a}(\bar{u}(t), \nabla \bar{u}(t)) \in L^{\infty}\left(\mathbb{R}^{N}\right)$ for a.e. $t \in(0, \tau)$

or

(b) $\bar{u}(t) \in B V_{\text {loc }}\left(\mathbb{R}^{N}\right)$ for a.e. $t \in(0, \tau)$ and $\operatorname{supp} u \cap\left([0, \tau] \times \mathbb{R}^{N}\right)$ is compact, then $\bar{u}(t) \geq u(t)$ for all $t \in(0, \tau)$.

Proof. Case (a) is proved in [5]. Indeed, it is easy to check that, up to redefining $\mathbf{a}(z, \mathbf{v})=\mathbf{a}(-z, \mathbf{v})$ for $z<0$, Assumption 1.1 implies all the assumptions of [5, Theorem 2] except for (H1) (cf. Theorem 2.2 above) and for two regularity properties of $u$ (namely, $\operatorname{div} \mathbf{a}(u(t), \nabla u(t)) \in\left(B V\left(\mathbb{R}^{N}\right)\right)^{*}$ for a.e. $t$ and $u_{t} \in$ $\left.\left(L_{\text {loc }}^{1}\left([0,+\infty) ; B V_{2}\left(\mathbb{R}^{N}\right)\right)\right)^{*}\right)$. However, following the proof one sees that the properties of $D_{0}$ are only used for $|z| \in[a, b]$ with $0<a<b<\infty$, hence (H1) is not used, and that the two additional regularity properties of $u$ are not used at all. Hence the same proof applies under Assumption 1.1.

For case (b), we note that the proof of [5, Theorem 2] applies Kruzkhov's doubling argument, using (2.8) for both $\bar{u}$ (in $(s, y)$-variables) and $u$ (in $(t, x)$-variables), with testing functions

$$
\eta_{m, n}(t, x, s, y)=\rho_{m}(x-y) \tilde{\rho}_{n}(t-s) \phi\left(\frac{t+s}{2}\right),
$$


where $\rho_{m}, \rho_{n}$ are standard mollifiers and $\phi$ is a nonnegative smooth function with compact support in $(0, \tau)$. The proof of (b) follows this argument very closely, hence we only highlight the main changes. We add a cutoff in space, i.e., we use testing functions

$$
\eta_{m, n}(t, x, s, y)=\rho_{m}(x-y) \tilde{\rho}_{n}(t-s) \phi\left(\frac{t+s}{2}\right) \psi\left(\frac{x+y}{2}\right),
$$

where $\psi$ is a nonnegative smooth function with compact support in $\mathbb{R}^{N}$. In this way, the arguments in [5, proof of Theorem 2] may be redone assuming only local integrability of $\bar{u}$. Passing to the limit as $m, n \rightarrow+\infty$, one finds that

$$
-\int_{0}^{\tau} \int_{\mathbb{R}^{N}}(u-\bar{u})_{+} \psi \phi_{t} \mathrm{~d} x \mathrm{~d} t \leq-\int_{0}^{\tau} \int_{\mathbb{R}^{N}} \phi \chi_{\{u>\bar{u}\}}(\mathbf{a}(u, \nabla u)-\mathbf{a}(\bar{u}, \nabla \bar{u})) \cdot \nabla \psi \mathrm{d} x \mathrm{~d} t .
$$

Since $\operatorname{supp}(u) \cap\left([0, \tau] \times \mathbb{R}^{N}\right)$ is compact, $(u-\bar{u})_{+}=0$ and $\chi_{\{u>\bar{u}\}}=0$ in $[0, \tau] \times\{|x| \geq$ $R\}$ for $R$ sufficiently large. Hence, for any $\psi$ such that $\psi \equiv 1$ for $|x| \leq R$, we obtain

$$
-\int_{0}^{\tau} \int_{\mathbb{R}^{N}}(u-\bar{u})_{+} \psi \phi_{t} \mathrm{~d} x \mathrm{~d} t \leq 0
$$

and the conclusion follows from the arbitrariness of $\phi$ and $\psi$.

3. The finite speed of propagation property. In this section we prove Theorem 1.2 by a generalization of techniques from [5].

Proof of Theorem 1.2. Let $C=\operatorname{supp}\left(u_{0}\right)$ and let $C(s):=C+B(0, s)$. We will argue below that

$$
\bar{u}(t, x)=\beta \chi_{C(v t)}, \quad v:=\operatorname{esssup}_{z \in(0, \beta)} \varphi^{\prime}(z)
$$

is a supersolution to $(1.1)$ in $(0, \tau) \times \mathbb{R}^{N}$ for all $\tau>0$ and all $\beta>0$. Choosing $\beta=\left\|u_{0}\right\|_{\infty}$ we also have $\bar{u}(0) \geq u_{0}$ : hence Theorem 1.2 follows from part (a) of Theorem 2.5.

We note that

$$
\mathbf{a}(\bar{u}, \nabla \bar{u}) \equiv 0 .
$$

We also note that the function $d_{C}(x):=\operatorname{dist}(x, C)$ is Lipschitz continuous in $\mathbb{R}^{N}$ with $\left|\nabla d_{C}(x)\right|=1$ for a.e. $x \in \mathbb{R}^{N} \backslash C$ (see, e.g., [18, proof of Lemma 3.2.34]). Furthermore, one easily checks that $\left\{x \in \mathbb{R}^{N}: d_{C}(x)=s\right\}=\partial C(s)$ for all $s>0$. Therefore $\partial C(s)$ is $\mathcal{H}^{N-1}$-measurable for a.e. $s>0$ (see [17, Lemma 2 in section 3.4.1]). We claim that

$$
\partial_{t} \chi_{C(v t)}=v \mathcal{H}^{N-1}\llcorner\partial C(v t)
$$

in the sense that

$$
\int_{0}^{+\infty} \int_{\mathbb{R}^{N}} \chi_{C(v t)} \phi_{t} \mathrm{~d} x \mathrm{~d} t=-v \int_{0}^{+\infty} \int_{\partial C(v t)} \phi \mathrm{d} \mathcal{H}^{N-1} \mathrm{~d} t
$$

for any smooth function $\phi$ with compact support in $(0, \infty) \times \mathbb{R}^{N}$. We fix one of such $\phi$ and, for $h>0$ so small that $\phi=0$ on $(0,2 h) \times \mathbb{R}^{N}$, we compute

$$
\begin{aligned}
I_{h} & :=\int_{0}^{+\infty} \int_{\mathbb{R}^{N}} \chi_{C(v t)}(x) \frac{\phi(t+h, x)-\phi(t, x)}{h} \mathrm{~d} x \mathrm{~d} t \\
& =-\int_{h}^{+\infty} \int_{\mathbb{R}^{N}} \phi(t, x) \frac{\chi_{C(v t)}(x)-\chi_{C(v(t-h))}(x)}{h} \mathrm{~d} x \mathrm{~d} t .
\end{aligned}
$$


Applying a corollary of the coarea formula, namely, [17, Theorem 2 in section 3.4.3], we obtain

$$
I_{h}=-\frac{1}{h} \int_{h}^{+\infty} \int_{v(t-h)}^{v t} \int_{\partial C(s)} \phi(t, x) \mathrm{d} \mathcal{H}^{N-1} \mathrm{~d} s \mathrm{~d} t .
$$

Exchanging the order of integration (recall that $\operatorname{supp} \phi$ is compact and $\phi=0$ in $\left.(0,2 h) \times \mathbb{R}^{N}\right)$ and letting $\bar{s}=s / v$, we find

$$
I_{h}=-v \int_{h}^{+\infty} \int_{\partial C(v \bar{s})} \frac{1}{h} \int_{\bar{s}}^{\bar{s}+h} \phi(t, x) \mathrm{d} t \mathrm{~d} \mathcal{H}^{N-1} \mathrm{~d} \bar{s}
$$

hence (3.3) follows passing to the limit as $h \rightarrow 0$.

To complete the proof, take any $\tau>0, S \in \mathcal{T}^{-}, T \in \mathcal{T}^{+}$, and any nonnegative smooth function $\phi$ with compact support in $(0, \tau) \times \mathbb{R}^{N}$. Arguing exactly as in the proof of [5, Proposition 1, section 5.1], we obtain for the measure on the left-hand side of (2.8) that

$$
h_{S}(\bar{u}(t), D T(\bar{u}(t)))+h_{T}(\bar{u}(t), D S(\bar{u}(t)))=J_{(T S)^{\prime} \varphi}(\beta) \mathcal{H}^{N-1}\llcorner\partial C(v t)
$$

for a.e. $t>0$. Now,

$$
J_{(T S)^{\prime} \varphi}(\beta) \stackrel{(2.1)}{=}(T S \varphi)(\beta)-(T S \varphi)(0)-\int_{0}^{\beta} T(r) S(r) \varphi^{\prime}(r) \mathrm{d} r .
$$

Since $\varphi(0)=0, \varphi \geq 0, S \in \mathcal{T}^{-}$, and $T \in \mathcal{T}^{+}$, we have $(T S \varphi)(\beta) \leq 0,(T S \varphi)(0)=0$, and, recalling $(3.1),-T(r) S(r) \varphi^{\prime}(r) \leq-T(r) S(r) v$ for any $r \in(0, \beta)$. Therefore

$$
J_{(T S)^{\prime} \varphi}(\beta) \leq-v \int_{0}^{\beta} T(r) S(r) \mathrm{d} r \stackrel{(2.1)}{=}-v J_{T S}(\beta) .
$$

Combining (3.4) and (3.5), we obtain for the left-hand side of (2.8)

$$
\begin{aligned}
& \int_{0}^{+\infty}\left\langle h_{S}(\bar{u}, D T(\bar{u}))+h_{T}(\bar{u}, D S(\bar{u})), \phi\right\rangle \mathrm{d} t \\
& \leq-v J_{T S}(\beta) \int_{0}^{+\infty} \int_{\partial C(v t)} \phi \mathrm{d} \mathcal{H}^{N-1} \mathrm{~d} t .
\end{aligned}
$$

On the other hand, the right-hand side of (2.8) is given by

$$
\begin{aligned}
& \int_{0}^{+\infty} \int_{\mathbb{R}^{N}}\left(J_{T S}(\bar{u}) \phi_{t}-T(\bar{u}) S(\bar{u}) \mathbf{a}(\bar{u}, \nabla \bar{u}) \cdot \nabla \phi\right) \mathrm{d} x \mathrm{~d} t \\
& \stackrel{(3.2)}{=} \int_{0}^{+\infty} \int_{\mathbb{R}^{N}} J_{T S}(\bar{u}) \phi_{t} \mathrm{~d} x \mathrm{~d} t \stackrel{(3.1)}{=} J_{T S}(\beta) \int_{0}^{+\infty} \int_{\mathbb{R}^{N}} \chi_{C(v t)} \phi_{t} \mathrm{~d} x \mathrm{~d} t \\
& \stackrel{(3.3)}{=}-v J_{T S}(\beta) \int_{0}^{+\infty} \int_{\partial C(v t)} \phi \mathrm{d} \mathcal{H}^{N-1} \mathrm{~d} t .
\end{aligned}
$$

Combining (3.6) and (3.7), we see that (2.8) holds. Hence $\bar{u}$ is a supersolution. 
4. The waiting time phenomenon. In this section we prove Theorem 1.4 and Corollary 1.5. We split the proof of Theorem 1.4 for the two equations.

Proof of Theorem 1.4, (1.3). We recall (1.3) and (1.10):

$$
u_{t}-\operatorname{div} \mathbf{a}_{1}(u, \nabla u)=0, \quad \mathbf{a}_{1}(z, \mathbf{v})= \begin{cases}\frac{z^{m} \mathbf{v}}{\sqrt{z^{2}+|\mathbf{v}|^{2}}} & \text { if }(z, \mathbf{v}) \neq(0, \mathbf{0}), \\ 0 & \text { if }(z, \mathbf{v})=(0, \mathbf{0}) .\end{cases}
$$

Up to a translation in space, we may assume without losing generality that $x_{0}=0$.

For $A, \alpha, \beta \in(0, \infty)$, let

$$
\bar{u}(t, x):=A(\tau-t)^{-\beta} r^{\alpha}, \quad r:=|x| .
$$

We will choose $A, \alpha$, and $\beta$ such that

$$
\mathcal{F}[\bar{u}]:=\bar{u}_{t}-\operatorname{div} \mathbf{a}_{1}(\bar{u}, \nabla \bar{u}) \geq 0 \quad \text { for all } t \in(0, \tau), r>0 .
$$

We note that

$$
\mathcal{F}[\bar{u}]=A \beta(\tau-t)^{-\beta-1} r^{\alpha}-A^{m}(\tau-t)^{-m \beta} \operatorname{div} \mathbf{a}_{1}\left(r^{\alpha}, \nabla r^{\alpha}\right) .
$$

Hence, choosing $\beta=1 /(m-1)$,

$$
\mathcal{F}[\bar{u}]=\frac{A}{m-1}(\tau-t)^{-\frac{m}{m-1}}\left(r^{\alpha}-(m-1) A^{m-1} \operatorname{div} \mathbf{a}_{1}\left(r^{\alpha}, \nabla r^{\alpha}\right)\right) .
$$

Using

$$
\nabla r^{\alpha}=\alpha r^{\alpha-2} x, \quad\left|\nabla r^{\alpha}\right|^{2}=\alpha^{2} r^{2 \alpha-2}, \quad \nabla r \cdot x=r, \quad \operatorname{div} x=N,
$$

we compute

$$
\begin{aligned}
\mathbf{a}_{1}\left(r^{\alpha}, \nabla r^{\alpha}\right) & =\frac{r^{\alpha m} \nabla r^{\alpha}}{\sqrt{r^{2 \alpha}+\left|\nabla r^{\alpha}\right|^{2}}}=\frac{r^{\alpha m-1} x}{\sqrt{1+\frac{r^{2}}{\alpha^{2}}}} \\
\operatorname{div} \mathbf{a}_{1}\left(r^{\alpha}, \nabla r^{\alpha}\right) & =\frac{\mathrm{d}}{\mathrm{d} r}\left(\frac{r^{\alpha m-1}}{\sqrt{1+\frac{r^{2}}{\alpha^{2}}}}\right) \nabla r \cdot x+\frac{r^{\alpha m-1}}{\sqrt{1+\frac{r^{2}}{\alpha^{2}}}} \operatorname{div} x \\
& =\frac{r^{\alpha m-1}}{\left(1+\frac{r^{2}}{\alpha^{2}}\right)^{3 / 2}}\left(\alpha m+N-1+(\alpha m+N-2) \frac{r^{2}}{\alpha^{2}}\right) .
\end{aligned}
$$

Inserting (4.4) into (4.2) and choosing $\alpha=1 /(m-1)$, we see that

$$
\begin{aligned}
\mathcal{F}[\bar{u}] & =\frac{A}{m-1}(\tau-t)^{-\frac{m}{m-1}} r^{\frac{1}{m-1}}\left(1-A^{m-1} f\left((m-1)^{2} r^{2}\right)\right), \\
f(s) & :=\frac{m+(N-1)(m-1)+(m+(N-2)(m-1))|s|}{(1+|s|)^{3 / 2}} .
\end{aligned}
$$

Note that $\|f\|_{\infty}=f(0)=m+(N-1)(m-1)$. Hence, choosing $A=A_{m}:=$ $(m+(N-1)(m-1))^{-1 /(m-1)}$, we conclude that

$$
\bar{u}(t, x):=A_{m}(\tau-t)^{-\frac{1}{m-1}}|x|^{\frac{1}{m-1}}
$$

is such that (4.1) holds. 
We now show that the function $\bar{u}$ in (4.5) is a supersolution to $(1.3)$ in $(0, \tau) \times \mathbb{R}^{N}$ for all $\tau>0$ in the sense of Definition 2.4. Since $\bar{u} \in C\left((0, \tau) ; W_{l o c}^{1,1}\left(\mathbb{R}^{N}\right)\right), \bar{u}$ satisfies (i) in Definition 2.4. Since

$$
\mathbf{a}_{1}(\bar{u}, \nabla \bar{u}) \stackrel{(4.4)}{=} A_{m}(\tau-t)^{-\frac{1}{m-1}} \frac{|x|^{\frac{1}{m-1}} x}{\sqrt{1+(m-1)^{2}|x|^{2}}} \in L_{l o c}^{\infty}\left([0, \tau) \times \mathbb{R}^{N}\right),
$$

$\bar{u}$ satisfies (ii) in Definition 2.4. Hence, it remains to show that $\bar{u}$ fulfills inequality (2.8) for any $T \in \mathcal{T}^{+}, S \in \mathcal{T}^{-}$, and $\phi \in C_{c}^{\infty}\left((0, \tau) \times \mathbb{R}^{N}\right)$. Since $\bar{u}(t) \in W^{1,1}\left(\mathbb{R}^{N}\right)$ for all $t \in(0, \tau),(2.4)-(2.7)$ imply that

$$
\left\langle h_{S}(\bar{u}, D T(\bar{u}))+h_{T}(\bar{u}, D S(\bar{u})), \phi\right\rangle=\int_{\mathbb{R}^{N}} \phi \mathbf{a}_{1}(\bar{u}, \nabla \bar{u}) \cdot \nabla(S(\bar{u}) T(\bar{u})) \mathrm{d} x,
$$

hence inequality $(2.8)$ reduces to

$$
\begin{aligned}
& \int_{0}^{\infty} \int_{\mathbb{R}^{N}} \phi \mathbf{a}_{1}(\bar{u}, \nabla \bar{u}) \cdot \nabla(T(\bar{u}) S(\bar{u})) \mathrm{d} x \mathrm{~d} t \\
& \leq \int_{0}^{\infty} \int_{\mathbb{R}^{N}}\left(J_{T S}(\bar{u}) \phi_{t}-T(\bar{u}) S(\bar{u}) \mathbf{a}_{1}(\bar{u}, \nabla \bar{u}) \cdot \nabla \phi\right) \mathrm{d} x \mathrm{~d} t .
\end{aligned}
$$

In order to prove (4.7), we start from (4.1): since $T \geq 0, \phi \geq 0$, and $S \leq 0$,

$$
0 \geq \int_{0}^{\infty} \int_{\mathbb{R}^{N} \backslash B_{\varepsilon}(0)} \phi T(\bar{u}) S(\bar{u})\left(\bar{u}_{t}-\operatorname{div} \mathbf{a}_{1}(\bar{u}, \nabla \bar{u})\right) \mathrm{d} x \mathrm{~d} t
$$

for all $\varepsilon>0$, where $B_{\varepsilon}(0)=\left\{x \in \mathbb{R}^{N}:|x|<\varepsilon\right\}$. Integrating by parts in (4.8), we obtain

$$
\begin{aligned}
& \int_{0}^{\infty} \int_{\mathbb{R}^{N} \backslash B_{\varepsilon}(0)} \phi \mathbf{a}_{1}(\bar{u}, \nabla \bar{u}) \cdot \nabla(T(\bar{u}) S(\bar{u})) \mathrm{d} x \mathrm{~d} t \\
& \leq \int_{0}^{\infty} \int_{\partial\left(\mathbb{R}^{N} \backslash B_{\varepsilon}(0)\right)} \phi T(\bar{u}) S(\bar{u}) \mathbf{a}_{1}(\bar{u}, \nabla \bar{u}) \cdot \mathbf{n} \mathrm{d} \mathcal{H}^{N-1} \mathrm{~d} t \\
& \quad+\int_{0}^{\infty} \int_{\mathbb{R}^{N} \backslash B_{\varepsilon}(0)}\left(J_{T S}(\bar{u}) \phi_{t}-T(\bar{u}) S(\bar{u}) \mathbf{a}_{1}(\bar{u}, \nabla \bar{u}) \cdot \nabla \phi\right) \mathrm{d} x \mathrm{~d} t .
\end{aligned}
$$

The passage to the limit as $\varepsilon \rightarrow 0$ in (4.9) is straightforward: in view of (4.6) and dominated convergence, the bulk integrals converge to their counterpart in $\mathbb{R}^{N}$ and the boundary integral vanishes. Therefore (2.8) holds and $\bar{u}$ is a supersolution.

The statement now follows immediately from part (b) of Theorem 2.5. Indeed, in view of (1.15), $C>0$ exists such that $u_{0}(x) \leq C|x|^{1 /(m-1)}$ for a.e. $x \in \mathbb{R}^{N}$. Choosing $\tau=\tau_{*}:=\left(C / A_{m}\right)^{1-m}$ in (4.5), we see that

$$
u_{0}(x) \leq C|x|^{\frac{1}{m-1}}=A_{m} \tau^{-\frac{1}{m-1}}|x|^{\frac{1}{m-1}}=\bar{u}(0, x) \quad \text { for a.e. } x \in \mathbb{R}^{N},
$$

hence $u \leq \bar{u}$ in $(0, \tau) \times \mathbb{R}^{N}$ : since $A_{m}=C \tau_{*}^{1 /(m-1)}$, we obtain (1.17).

Proof of Theorem 1.4, (1.4). We recall (1.4) and (1.11):

$$
u_{t}-\operatorname{div} \mathbf{a}_{2}(u, \nabla u)=0, \quad \mathbf{a}_{2}(z, \mathbf{v})= \begin{cases}\frac{(M-1) z^{M-1} \mathbf{v}}{\sqrt{1+(M-1)^{2} z^{2 M-4}|\mathbf{v}|^{2}}} & \text { if } z>0 \\ 0 & \text { if } z=0 .\end{cases}
$$


Up to a translation in space, we may assume without losing generality that $x_{0}=0$. For $A, \alpha, \beta \in(0, \infty)$, let

$$
\bar{u}(t, x):=A(\tau-t)^{-\beta} r^{\alpha}, \quad r:=|x| .
$$

As above, choosing $\beta=1 /(M-1)$, for $r>0$ we obtain

$$
\mathcal{F}[\bar{u}]:=\bar{u}_{t}-\operatorname{div} \mathbf{a}_{2}(\bar{u}, \nabla \bar{u})
$$

$$
=\frac{A}{M-1}(\tau-t)^{-\frac{M}{M-1}}\left(r^{\alpha}-(M-1)^{2} A^{M-1} \operatorname{div}\left(\frac{r^{\alpha(M-1)} \nabla r^{\alpha}}{\sqrt{1+K^{2}(t, r)}}\right)\right),
$$

where

$$
\begin{aligned}
K^{2}(t, r) & :=A^{2(M-1)}(M-1)^{2}(\tau-t)^{-2} r^{2 \alpha(M-2)}\left|\nabla r^{\alpha}\right|^{2} \\
& \stackrel{(4.3)}{=} A^{2(M-1)}(M-1)^{2} \alpha^{2}(\tau-t)^{-2} r^{2(\alpha(M-1)-1)} .
\end{aligned}
$$

Using (4.3) and arguing as in (4.4), we compute

$$
\begin{aligned}
\operatorname{div}\left(\frac{r^{\alpha(M-1)} \nabla r^{\alpha}}{\sqrt{1+K^{2}(t, r)}}\right) & =\operatorname{div}\left(\frac{\alpha r^{\alpha M-2} x}{\sqrt{1+K^{2}(t, r)}}\right) \\
& =\alpha r^{\alpha M-2} \frac{\alpha M+N-2+(\alpha+N-1) K^{2}(t, r)}{\left(1+K^{2}(t, r)\right)^{3 / 2}} .
\end{aligned}
$$

Inserting (4.11) into (4.10) and choosing $\alpha=2 /(M-1)$, we obtain

$$
\begin{aligned}
\mathcal{F}[\bar{u}] & =\frac{A}{(M-1)}(\tau-t)^{-\frac{M}{M-1}} r^{\frac{2}{M-1}}\left(1-2 A^{M-1} g\left(K^{2}(t, r)\right)\right), \\
g(s) & :=\frac{N(M-1)+2+((N-1)(M-1)+2)|s|}{(1+|s|)^{3 / 2}} .
\end{aligned}
$$

Note that $\|g\|_{\infty}=g(0)=N(M-1)+2$. Hence, choosing $A=A_{M}:=(2 N(M-1)+$ $4)^{-1 /(M-1)}$, we conclude that $\mathcal{F}[\bar{u}] \geq 0$ for all $t \in(0, \tau)$ and all $r>0$. From here on, the proof is completely analogous to the one for (1.3) and therefore we omit it.

We conclude with the proof of Corollary 1.5.

Proof of Corollary 1.5. We look only at (1.3), the proof for (1.4) being completely analogous. We denote by $\left\{e_{1}, \ldots, e_{N}\right\}$ the canonical basis of $\mathbb{R}^{N}$ and we let $x_{i}=x \cdot e_{i}$. Up to a translation and a rotation in space, we may assume without losing generality that $0=x_{0} \in \partial \operatorname{supp}\left(u_{0}\right)$ and that $w=e_{1}$, so that the exterior cone property becomes

$$
\operatorname{supp}\left(u_{0}\right) \cap \mathcal{C}_{B_{0}}=\emptyset, \quad \mathcal{C}_{B_{0}}:=\left\{x \in \mathbb{R}^{N}: x_{1}<-B_{0}|\underline{x}|\right\}, \quad \underline{x}:=x-x_{1} e_{1} .
$$

If $N=1$ the proof is straightforward: we have $\mathcal{C}_{B_{0}}=(-\infty, 0)$ (for any $B_{0}$ ) and

$$
u_{0}(x) \stackrel{(1.15),(4.12)}{\leq} C x_{+}^{\frac{1}{m-1}} \leq C|x-z|^{\frac{1}{m-1}} \text { for all } z \leq 0 \text { and a.e. } x \in \mathbb{R} .
$$

Hence Theorem 1.4 may be applied with $x_{0}=z$ for all $z \leq 0$, leading to $\operatorname{supp}(u(t, \cdot)) \subseteq$ $[0, \infty)$ for all $t<\left(C / A_{m}\right)^{1-m}$.

If $N>1$, an elementary constrained minimization shows, for any $B>0$, that

$$
\operatorname{dist}\left(x, \mathcal{C}_{B}\right)=\left\{\begin{array}{ll}
|x| & \text { if } x_{1} \geq|\underline{x}| / B \\
\frac{x_{1}+B|\underline{x}|}{\sqrt{1+B^{2}}} & \text { if } x_{1}<|\underline{x}| / B
\end{array} \quad \text { for all } x \in \mathbb{R}^{N} \backslash \mathcal{C}_{B} .\right.
$$


On the other hand, a simple computation shows that

$$
|x| \leq x_{1}+B|\underline{x}| \quad \text { for all } x \in \mathbb{R}^{N} \backslash \mathcal{C}_{B_{0}}
$$

for all $B$ such that $B^{2}-2 B_{0} B-1 \geq 0$, in particular, for $B=B_{1}:=B_{0}+\sqrt{1+B_{0}^{2}}$. Therefore, for all $x \in \mathbb{R}^{N} \backslash \mathcal{C}_{B_{0}}$ we have

$$
\begin{aligned}
& |x| \stackrel{(4.13)}{=} \operatorname{dist}\left(x, \mathcal{C}_{B_{1}}\right) \quad \text { if } x_{1} \geq|\underline{x}| / B_{1}, \\
& |x| \stackrel{(4.14)}{\leq} \quad x_{1}+B_{1}|\underline{x}| \stackrel{(4.13)}{=} \sqrt{1+B_{1}^{2}} \operatorname{dist}\left(x, \mathcal{C}_{B_{1}}\right) \quad \text { if } x_{1}<|\underline{x}| / B_{1} \text {, }
\end{aligned}
$$

i.e.,

$$
|x| \leq \sqrt{1+B_{1}^{2}}|x-z| \quad \text { for all } x \in \mathbb{R}^{N} \backslash \mathcal{C}_{B_{0}}, z \in \mathcal{C}_{B_{1}} .
$$

Taking into account (4.12), (1.15) and (4.15) imply that

$$
u_{0}(x) \leq C \sqrt{1+B_{1}^{2}}|x-z|^{\frac{1}{m-1}} \quad \text { for all } z \in \mathcal{C}_{B_{1}} \text { and a.e. } x \in \mathbb{R}^{N} .
$$

Applying Theorem 1.4 with $x_{0}=z$ for all $z \in \mathcal{C}_{B_{1}}$, we conclude that

$$
\operatorname{supp}(u(t, \cdot)) \cap \mathcal{C}_{B_{1}}=\emptyset \quad \text { for all } t<\tau_{*}=\left(C \sqrt{1+B_{1}^{2}} / A_{m}\right)^{1-m},
$$

hence $0 \in \overline{\mathbb{R}^{N} \backslash \operatorname{supp}(u(t, \cdot))}$ for all $t<\left(C \sqrt{1+B_{1}^{2}} / A_{m}\right)^{1-m}$.

5. Heuristics. We present a heuristic argument, based on the hyperbolic character of the equations, in favor of the optimality of the exponents obtained in Theorem 1.4. Throughout the section we assume that

$$
u_{0}(x) \sim A x_{+}^{\alpha} \quad \text { in a neighborhood of } x=0
$$

and that the waiting time, denoted by $\tau_{*}$, is positive:

$$
\tau_{*}:=\sup \{\tau \geq 0: \operatorname{supp}(u) \cap([0, \tau] \times(-\infty, 0))=\emptyset\}>0 .
$$

As proved in $[11,12]$, as soon as a jump discontinuity forms at $x=0$, the front $s_{-}(t)$ (i.e., the left endpoint of $\operatorname{supp}(u(t, \cdot)))$ will start moving with a velocity given by a Rankine-Hugoniot relation:

$$
\frac{\mathrm{d} s_{-}(t)}{\mathrm{d} t}=-\left(u_{+}\left(t, s_{-}(t)\right)\right)^{m-1} \text { if } u \text { solves (1.3), } \quad \frac{\mathrm{d} s_{-}(t)}{\mathrm{d} t}=-1 \text { if } u \text { solves (1.4). }
$$

Therefore $u(t, \cdot)$ must be continuous at $x=0$ for $t \in\left[0, \tau_{*}\right)$. We also assume that $u(t, \cdot)$ is monotone and continuously differentiable in an open right-neighborhood of $x=0$.

5.1. Equation (1.3). We argue for $t \in\left[0, \tau_{*}\right)$. Since $u(t, \cdot)$ is monotone and continuously differentiable in an open right-neighborhood of $x=0$, we have $u(t, \cdot) \ll$ $u_{x}(t, \cdot)$ in a right-neighborhood of $x=0$. Therefore $\sqrt{u^{2}+u_{x}^{2}} \sim u_{x}$, and we have

$$
u_{t} \sim\left(u^{m}\right)_{x}=m u^{m-1} u_{x}
$$

in a right-neighborhood of $x=0$. Let us now use the method of characteristics. For $\eta \geq 0$, let $X_{\eta}(t)$ be such that $U(t):=u\left(t, X_{\eta}(t)\right)=\eta$. We have

$$
0=\frac{\mathrm{d} U}{\mathrm{~d} t}=u_{t}+u_{x} \frac{\mathrm{d} X_{\eta}}{\mathrm{d} t} \sim u_{x}\left(m u^{m-1}+\frac{\mathrm{d} X_{\eta}}{\mathrm{d} t}\right), \quad \text { i.e., } \quad \frac{\mathrm{d} X_{\eta}}{\mathrm{d} t} \sim-m \eta^{m-1}
$$


The initial condition (5.1) yields

$$
A X_{\eta}(0)^{\alpha} \sim u\left(0, X_{\eta}(0)\right)=\eta \text {, i.e., } X_{\eta}(0)=(\eta / A)^{1 / \alpha} .
$$

It follows from (5.5)-(5.6) that the characteristics are (at leading order) given by

$$
X_{\eta}(t) \sim(\eta / A)^{1 / \alpha}-m \eta^{m-1} t .
$$

Now, two characteristics with values $\eta$ and $\xi$ intersect if and only if

$$
(\eta / A)^{1 / \alpha}-m \eta^{m-1} t=(\xi / A)^{1 / \alpha}-m \xi^{m-1} t, \quad \text { i.e., } \quad m A^{1 / \alpha} t=\frac{\eta^{1 / \alpha}-\xi^{1 / \alpha}}{\eta^{m-1}-\xi^{m-1}},
$$

and we have

$$
\lim _{(\xi, \eta) \rightarrow(0,0)} \frac{\eta^{1 / \alpha}-\xi^{1 / \alpha}}{\eta^{m-1}-\xi^{m-1}}= \begin{cases}0 & \text { if } \alpha<\frac{1}{m-1} \\ 1 & \text { if } \alpha=\frac{1}{m-1} \\ +\infty & \text { if } \alpha>\frac{1}{m-1}\end{cases}
$$

For $\alpha \geq 1 /(m-1),(5.7)-(5.8)$ show that, for short times, the characteristics emanating from a right-neighborhood of $x=0$ do not intersect; in particular, no discontinuity forms and no information travels through the characteristic $X_{0}(t)=0$, which is consistent with a positive waiting time. Vice versa, for $\alpha<1 /(m-1)$ the characteristics emanating from a right-neighborhood of $x=0$ instantaneously intersect, in contradiction with the continuity of $u$ (hence, with a positive waiting time).

In the case $\alpha<1 /(m-1)$, the instantaneous intersection of characteristics implies that a shock instantaneously forms at $x=s_{-}(0)=0$. As proved in [11, section 8], the graph of $u$ has a vertical contact angle at the shock: $u_{x}(t, x) \rightarrow+\infty$ as $x \rightarrow s_{-}(t)^{+}$. Hence (5.4) remains valid in a right-neighborhood of $x=s_{-}(t)$, and we may compute the short-time evolution of the front, $x=s_{-}(t)$, by solving the caustic system

$$
\left\{\begin{array}{l}
0=F(t, x, \eta):=x-\left((\eta / A)^{1 / \alpha}-m \eta^{m-1} t\right), \\
0=\frac{\partial F}{\partial \eta}=-\frac{1}{\alpha A^{1 / \alpha}} \eta^{1 / \alpha-1}+m(m-1) \eta^{m-2} t .
\end{array}\right.
$$

Letting $a:=1-\alpha(m-1)>0$, the solution is given by

$$
\eta=\left(m(m-1) \alpha A^{1 / \alpha} t\right)^{\frac{\alpha}{a}}, \quad x=-a\left(m A^{m-1}(1-a)^{1-a} t\right)^{\frac{1}{a}} .
$$

Hence we recover the shock's position at leading order for $t \ll 1$ :

$$
s_{-}(t) \sim-a\left(m A^{m-1}(1-a)^{1-a} t\right)^{\frac{1}{a}} \quad \text { for } t \ll 1 .
$$

The height of the shock, $u_{+}\left(t, s_{-}(t)\right)$, can be recovered through (5.3):

$$
u_{+}\left(t, s_{-}(t)\right)=\left(-\frac{\mathrm{d} s_{-}(t)}{\mathrm{d} t}\right)^{\frac{1}{m-1}}=\left(m A^{m-1}\right)^{\frac{1}{a(m-1)}}((1-a) t)^{\frac{1-a}{a(m-1)}} .
$$

Remark 5.1. Rather than using the method of characteristics, the heuristic in [9] fixes the domain, passing to Lagrangian coordinates. It is argued that the waiting time $\tau_{*}$ is characterized by $u_{+}\left(\tau_{*}, s_{-}\left(\tau_{*}\right)\right)=0$ and $u_{+}\left(t, s_{-}(t)\right)>0$ for $t>\tau_{*}$. This characterization, which is fully consistent with our argument above, is then translated into the condition $\left.\frac{\mathrm{d}}{\mathrm{d} t} u_{+}\left(t, s_{-}(t)\right)\right|_{t=\tau_{*}}>0$, leading to $\alpha \leq 1 / m$ : such translation does not seem to be optimal, since $u_{+}\left(t, s_{-}(t)\right)$ may become positive after $\tau_{*}$ even if $\left.\frac{\mathrm{d}}{\mathrm{d} t} u_{+}\left(t, s_{-}(t)\right)\right|_{t=\tau_{*}}=0$. Note that the two arguments are consistent, in the sense that (5.10) also yields $\left.\frac{\mathrm{d}}{\mathrm{d} t} u_{+}\left(t, s_{-}(t)\right)\right|_{t=\tau_{*}}>0$ if and only if $\alpha \leq 1 / m$. 
5.2. Equation (1.4). We argue for $t \in\left[0, \tau_{*}\right.$ ) (see (5.2)). We distinguish two cases:

(1) If $\left(\left(u^{M-1}(t, x)\right)_{x}\right)^{2} \gg 1$ in a right-neighborhood of $x=0$, at leading order (1.4) reads as

$$
u_{t} \sim-u_{x}
$$

whose characteristics have slope -1 , thus violating the assumption of a positive waiting time.

(2) If $\left(\left(u^{M-1}(t, x)\right)_{x}\right)^{2} \ll 1$ in a right-neighborhood of $x=0$, at leading order (1.4) reads as

$$
u_{t} \sim(M-1)\left(u^{M-1} u_{x}\right)_{x}
$$

hence the solutions to (1.4) locally behave as those of the porous medium equation, for which a waiting time phenomenon is known to occur if and only if $\alpha \geq 2 /(M-1)$.

Therefore a waiting time phenomenon can occur only if $\alpha \geq 2 /(M-1)$ (and $\left(\left(u^{M-1}\right)_{x}\right)^{2} \ll 1$ as $x \rightarrow 0^{+}$, which in this case is automatically satisfied).

Appendix. In this section we give details concerning the existence and uniqueness statements in Theorem 2.2.

The uniqueness part of Theorem 2.2 is proved in [3]. The argument is identical to that given in the proof of Theorem 2.5(a).

When (H1) holds, the existence part of Theorem 2.2 is proved in [3]. Indeed, it is easy to check that, up to redefining $\mathbf{a}(z, \mathbf{v})=\mathbf{a}(-z, \mathbf{v})$ for $z<0$, Assumption 1.1 and (H1) imply all the assumptions in [3, Theorem 4.5]. Note that the bounds $u \in L^{\infty}\left((0, \infty) \times \mathbb{R}^{N}\right)$ and $\mathbf{a}(u, \nabla u) \in L_{l o c}^{\infty}\left([0, \infty) \times \mathbb{R}^{N}\right)$ are not explicitly stated in [3, Theorem 4.5]; however, they are proved (see formulas (4.6), (4.10), and (4.22) in $[3])$.

When (H2) holds, the existence part of Theorem 2.2 follows from [12, Theorem 5.3], applied with $\bar{m}=1$ and $\Phi(u)=u$. Indeed, given $u_{0} \in L^{\infty}\left(\mathbb{R}^{N}\right) \cap L^{1}\left(\mathbb{R}^{N}\right)$ and $\mathbf{a}=\nabla_{\mathbf{v}} f$ satisfying Assumption 1.1, let $R_{0}>\left\|u_{0}\right\|_{\infty}$ and define

$$
\tilde{f}(z, \mathbf{v}):= \begin{cases}f(z, \mathbf{v}) & \text { if } 0 \leq z \leq R_{0} \\ f\left(R_{0}, \mathbf{v}\right) & \text { if } z>R_{0}\end{cases}
$$

One readily sees that $\tilde{\mathbf{a}}(z, \mathbf{v}):=\nabla_{\mathbf{v}} \tilde{f}(z, \mathbf{v})$ satisfies Assumption 1.1 and (H2) with constants independent of $R$. This implies that the assumptions of [12, Theorem 5.3] are satisfied: hence there exists an entropy solution $\tilde{u}$ of

$$
u_{t}=\operatorname{div} \tilde{\mathbf{a}}(u, \nabla u), \quad u(0, x)=u_{0}(x)
$$

in the sense of [12, Definition 5.1]. Now, in [12, Theorem 5.3] the bound $\|\tilde{u}\|_{\infty} \leq$ $\left\|u_{0}\right\|_{\infty}<R_{0}$ is proved, though not explicitly stated (see formulas (3.24) and (4.5) in [12]); in addition, the class of truncatures in [12, Definition 5.1] contains $\mathcal{T}^{+}$. Therefore $\tilde{u}$ is also an entropy solution of (1.1) in the sense of Definition 2.1. Since $\mathbf{a}(z, \mathbf{v}) \equiv \tilde{\mathbf{a}}(z, \mathbf{v})$ for $z \in\left[0, R_{0}\right]$, this yields the result.

Acknowledgments. The author acknowledges inspiring discussions with Juan Calvo and Salvador Moll. The author also thanks the referees for stimulating questions and useful comments. 


\section{REFERENCES}

[1] N. D. Alikakos, On the pointwise behavior of the solutions of the porous medium equation as $t$ approaches zero or infinity, Nonlinear Anal., 9 (1985), pp. 1095-1113.

[2] L. Ambrosio, N. Fusco, and D. Pallara, Functions of Bounded Variation and Free Discontinuity Problems, Oxford Math. Monogr., Oxford University Press, New York, 2000.

[3] F. Andreu, V. Caselles, and J. M. Mazón, The Cauchy problem for a strongly degenerate quasilinear equation, J. Eur. Math. Soc. (JEMS), 7 (2005), pp. 361-393.

[4] F. Andreu, V. Caselles, And J. M. Mazón, Some regularity results on the 'relativistic' heat equation, J. Differential Equations, 245 (2008), pp. 3639-3663.

[5] F. Andreu, V. Caselles, J. Mazón, and S. Moll, Finite propagation speed for limited flux diffusion equations, Arch. Ration. Mech. Anal., 182 (2006), pp. 269-297.

[6] F. Andreu, V. Caselles, J. M. Mazón, J. Soler, and M. Verbeni, Radially symmetric solutions of a tempered diffusion equation. A porous media, flux-limited case, SIAM J. Math. Anal., 44 (2012), pp. 1019-1049.

[7] J. Calvo, Analysis of a Class of Degenerate Parabolic Equations with Saturation Mechanisms, preprint.

[8] J. Calvo And V. Caselles, Local-in-time regularity results for some flux-limited diffusion equations of porous media type, Nonlinear Anal., 93 (2013), pp. 236-272.

[9] J. A. Carrillo, V. Caselles, and S. Moll, On the relativistic heat equation in one space dimension, Proc. Lond. Math. Soc., 107 (2013), pp. 1395-1423.

[10] V. Caselles, An existence and uniqueness result for flux limited diffusion equations, Discrete Contin. Dyn. Syst., 31 (2011), pp. 1151-1195.

[11] V. Caselles, On the entropy conditions for some flux limited diffusion equations, J. Differential Equations, 250 (2011), pp. 3311-3348.

[12] V. Caselles, Flux limited generalized porous media diffusion equations, Publ. Mat., 57 (2013), pp. $155-217$.

[13] V. CASElles, Convergence of flux limited porous media diffusion equations to its classical counterpart, Ann. Sc. Norm. Super. Pisa, in press (Doi: 10.2422/2036-2145.201204_004).

[14] G. DAL MASO, Integral representation on $B V(\Omega)$ of $\Gamma$-limits of variational integrals, Manuscripta Math., 30 (1980), pp. 387-416.

[15] R. Dal Passo, L. Giacomelli, and G. Grün, A waiting time phenomenon for thin film equations, Ann. Sc. Norm. Super. Pisa Cl. Sci. (4), 30 (2001), pp. 437-463.

[16] V. De Cicco, N. Fusco, and A. Verde, On $L^{1}$-lower semicontinuity in $B V(\Omega)$, J. Convex Anal., 12 (2005), pp. 173-185.

[17] L. C. Evans and R. F. Gariepy, Measure theory and fine properties of functions, Stud. Adv. Math., CRC Press, Boca Raton, FL, 1992.

[18] H. Federer, Geometric Measure Theory, Grundlehren Math. Wiss. 153, Springer-Verlag, New York, 1969

[19] J. FisheR, Upper bounds on waiting times for the thin-film equation: The case of weak slippage, Arch. Ration. Mech. Anal., 211 (2014), pp. 771-818.

[20] L. Giacomelli And G. Grün, Lower bounds on waiting times for degenerate parabolic equations and systems, Interfaces Free Bound., 8 (2006), pp. 111-129.

[21] G. Grün, Droplet spreading under weak slippage: The waiting time phenomenon, Ann. Inst. H. Poincaré Anal. Non Linéaire, 21 (2004), pp. 255-269. 